\title{
Signature Geochimique des Granitoides de la Branche Est du Sillon de Boundiali-Korhogo (Nord de la Cote D'ivoire)
}

\author{
Fofana Krohognon, \\ Allialy Marc Ephrem
}

Laboratoire de Géologie, Ressources Minérales et Energétiques (LGRME),

UFR Sciences de la Terre et des Ressources Minières (UFR-STRM),

Université Félix Houphouët-Boigny, Abidjan, Côte d’Ivoire

\section{Coulibaly Inza}

Laboratoire Géosciences et Environnement, UFR des Sciences et Gestion de l'Environnement (UFR-SGE), Université Nangui Abrogoua, Abidjan, Côte d'Ivoire

\section{Teha Koffi Raoul}

Laboratoire de Géologie, Ressources Minérales et Energétiques (LGRME),

UFR Sciences de la Terre et des Ressources Minières (UFR-STRM),

Université Félix Houphouët-Boigny, Abidjan, Côte d’Ivoire

Doi:10.19044/esj.2021.v17n34p223

Submitted: 24 June 2021

Accepted: 20 September 2021

Published: 30 September 2021
Copyright 2021 Author(s)

Under Creative Commons BY-NC-ND 4.0 OPEN ACCESS

Cite As:

Fofana K., Allialy M.E., Coulibaly I. \& Teha Koffi R. (2021). Signature Geochimique des Granitoides de la Branche Est du Sillon de Boundiali-Korhogo (Nord de la Cote D'ivoire). European Scientific Journal, ESJ, 17(34), 223.

https://doi.org/10.19044/esj.2021.v17n34p223

\section{Résumé}

Cet article résume les données relatives à la pétrographie et aux compositions en éléments majeurs et en traces des granitoïdes du sillon de Korhogo. Le sillon de Korhogo est situé au Nord de la Côte d'Ivoire, dans le domaine birimien. Ce sillon qui constitue la branche Est du sillon de Boundiali-Korhogo est à cheval sur les régions du Poro et du Tchologo. La méthodologie employée a permis à l'issue des observations macroscopiques, de recueillir des échantillons pour les études microscopiques et géochimiques. Les études pétrographiques indiquent que les granitoïdes du sillon de Korhogo sont des granodiorites et granites. Ces granitoïdes ont été affectés par une intense altération pervasive et fissurale témoignant ainsi de l'intensité de l'altération hydrothermale dans le sillon de Korhogo. Ces roches ont été 
affectées par un le métamorphisme de faciès schiste vert. Les données géochimiques montrent que les granitoïdes sont calco-alcalins, potassiques, métalumineux à peralumineux et de Type « $\mathrm{I}$ ». Ces roches sont enrichies en LREE [Granodiorite $\left((\mathrm{La} / \mathrm{Sm})_{N}=3,36-4,45 ;(\mathrm{Gd} / \mathrm{Yb})_{N}=1,56-2,51\right.$; et $\left.(\mathrm{La} / \mathrm{Yb})_{N}=7,29-20,92\right) ;$ Granite $\left((\mathrm{La} / \mathrm{Sm})_{N}=4,51-4,69 ;(\mathrm{Gd} / \mathrm{Yb})_{N}=1,00\right.$ 3,13 et $\left.(\mathrm{La} / \mathrm{Yb})_{N}=7,63-26,53\right]$ et présentent des anomalies négatives à quasiment nulles en cérium [Granite $\left(\mathrm{Ce} / \mathrm{Ce}^{*}=0,60-0,92\right)$; Granodiorite $(\mathrm{Ce} / \mathrm{Ce} *=0,80-1,01)]$. Les anomalies en europium varient des granodiorites $\left[\left(\mathrm{Eu} / \mathrm{Eu}^{*}=0,81-1,53\right)\right]$ aux granites [(Eu/Eu*= 0,90 - 1,11)]. Ces granitoïdes ont de faibles taux de $\mathrm{TiO}_{2}(<2 \%)$. Les arachnogrammes des éléments en trace normalisés au manteau primitif sont généralement enrichis en LILE (Cs, Ba, $\mathrm{Rb}, \mathrm{K}$ ) associés à une anomalie négative en $\mathrm{Nb}-\mathrm{Ta}$.

Les tendances géochimiques indiquent que les granitoïdes du sillon de Korhogo proviendraient d'un mélange crustal et mantellique et se seraient mis en place dans un contexte de subduction.

Mots clés: Birimien, Sillon de Korhogo, Granitoïde, Pétrographie, Géochimie

\section{Granitoids Geochemical Signature of the Boundiali-Korhogo Belt Eastern Branch (North of Cote D’ivoire)}

\section{Fofana Krohognon, \\ Allialy Marc Ephrem}

Laboratoire de Géologie, Ressources Minérales et Energétiques (LGRME),

UFR Sciences de la Terre et des Ressources Minières (UFR-STRM),

Université Félix Houphouët-Boigny, Abidjan, Côte d'Ivoire

\section{Coulibaly Inza}

Laboratoire Géosciences et Environnement, UFR des Sciences et Gestion de l'Environnement (UFR-SGE), Université Nangui Abrogoua, Abidjan, Côte d'Ivoire

\section{Teha Koffi Raoul}

Laboratoire de Géologie, Ressources Minérales et Energétiques (LGRME),

UFR Sciences de la Terre et des Ressources Minières (UFR-STRM),

Université Félix Houphouët-Boigny, Abidjan, Côte d'Ivoire

\section{Abstract}

This paper summarizes the data available on petrography and the concentrations of major and trace elements in granitoids from Korhogo greenstone belt province. The Korhogo greenstone belt is localed in the north of Côte d'Ivoire, in the birimian domain. This belt which constitutes the eastern branch of the Boundiali-Korhogo greenstone belt straddles the Poro and Tchologo areas. The methodology started by the macroscopic to 
microscopic observations then it continued to the geochemical studies. Petrographic studies indicate that the granitoids of Korhogo greenstone belt are granodiorites and granites. These granitoids were affected by intense pervasive and fissural alteration thus testifying the intensity of hydrothermal alteration in the Korhogo greenstone belt. These rocks were affected by regional greenschist facies. Geochemical datas show that the granitoids are calc-alkaline, potassic, metaluminous to peraluminous and "I" Type. These rocks are enriched in LREE [Granodiorite $(\mathrm{La} / \mathrm{Sm})_{N}=3,36-4,45 ;(\mathrm{Gd} / \mathrm{Yb})_{N}$ $=1,56-2,51$; et $\left.(\mathrm{La} / \mathrm{Yb})_{N}=7,29-20,92\right)$; Granite $(\mathrm{La} / \mathrm{Sm})_{N}=4,51-4,69$; $(\mathrm{Gd} / \mathrm{Yb})_{N}=1,00-3,13$ et $\left.(\mathrm{La} / \mathrm{Yb})_{N}=7,63-26,53\right]$ and present negative to non-exixting cerium anomalies [Granite (Ce / $\mathrm{Ce} *=0.60-0.92)$; Granodiorite $(\mathrm{Ce} / \mathrm{Ce} *=0.80-1.01)]$. The europium anomalies vary from granodiorites $[(\mathrm{Eu} / \mathrm{Eu} *=0.81-1.53)]$ to granites $[(\mathrm{Eu} / \mathrm{Eu} *=0.90-1.11)]$. These granitoids have low levels of $\mathrm{TiO}_{2}(<2 \%)$. Primitive mantle normalized, trace element patterns show that granitoids have geochemical patterns characterized by enrichment in LILE (Cs, Ba, Rb, K) associated with negative $\mathrm{Nb}-\mathrm{Ta}$ anomalies.

Geochemical trends indicate that the granitoids of Korhogo greenstone belt originate from a crustal and mantle mixture and were set up in a context of subduction.

Keywords: Birimian, Korhogo Greenstone Belt, Granitoids, Petrography, Geochemistry

\section{Introduction}

Les formations birimiennes sont composées de ceintures de roches vertes et de larges bassins sédimentaires, plus ou moins étirés dans la direction NE-SW qui contiennent des roches plutono-volcaniques, volcanoclastiques et sédimentaires, métamorphisées globalement dans les conditions du faciès des schistes verts et intrudés par des massifs de granitoïdes. Les ceintures de roches vertes birimiennes sont connues comme étant porteuses d'abondantes minéralisations aurifères (Milési et al., 1989) et la Côte d’Ivoire possède la plus grande part des formations birimiennes de l'Afrique de l'Ouest avec environ $35 \%$ de ces formations. Ces intrusions séparent et parfois recoupent les formations des ceintures et correspondent essentiellement à des tonalites, des granodiorites et des monzogranites. De manière schématique, on peut les classer en deux suites en fonction de leurs caractères pétrographiques, géochimiques et de leur âge de mise en place: i) une suite à caractère sodique représentée essentiellement par des granitoïdes à amphiboles définissant une série de type TTG mise en place entre 2,25 et 2,12 Ga; ii) une suite calcoalcaline à caractère potassique représentée par des granitoïdes à ortho et clinopyroxène, des granitoïdes à amphibole et des granitoïdes à biotite mis en 
place entre 2,12 et 2,09 Ga et parfois même une suite alcaline avec des granites et des syénites alcalins comme ceux décrits au Burkina Faso (Naba et al., 2004; Tapsoba et al., 2013), en Côte d'Ivoire (Morel et Alinat, 1993) et au Sénégal (Hirdes et Davis, 2002) mis en place à la fin de l'orogenèse entre 2,11 et 2,07 Ga. Les minéralisations aurifères dans le domaine paléoprotérozoïque sont souvent associées à des shear zones dans les zones de contact métasédiments - roches vertes/ intrusions granitoïdes (Milési et al., 1989 et 1992 ; Feybesse, 2001; Gbamélé, 2012 ; Houssou, 2013 ; Gnanzou, 2014 ; Ouattara, 2015). La ceinture de roches vertes de Boundiali-Korhogo est composée d'une branche Ouest orientée N-S appelée le sillon de Boundiali ou de la Bagoué et d'une branche Est, orientée NNE-SSO est appelée le sillon de Korhogo, qui appartient aux régions de Korhogo et du Tchologo. Les travaux scientifiques effectués, essentiellement lithologique (Turner, 1993 et Yacé, 2002) se résument au sillon de Boundiali. La branche Est qui constitue notre zone d'étude est sujet à de nombreuses activités minières qui ont conduit à la découverte du gisement de Tongon ainsi que la mise en évidence de plusieurs prospects prometteurs. Cependant très peu de travaux scientifiques ont été entrepris tant sur le plan pétrostructural, géochimique, que géochronologique. C'est dans ce contexte que cette étude a été initiée dans l'optique de contribuer à l'amélioration des connaissances pétrographiques et géochimiques sur les granitoïdes de Côte d'Ivoire, et en particulier ceux du sillon de Korhogo.

\section{Contexte géologique}

La Côte d'Ivoire appartient à la partie sud du Craton Ouest-Africain et plus précisément à la dorsale de Man ou dorsale de Léo (Fig. 1). Elle est constituée d'un socle précambrien qui représente $97,5 \%$ de sa superficie et d'un bassin sédimentaire côtier secondaire-tertiaire couvrant 2,5 \% du territoire. Ce socle d'âge précambrien comporte un domaine archéen (Kenema-Man) à l'ouest (3600-2500 Ma) et un domaine paléoprotérozoïque (Birimien) également dénommé domaine Baoulé-Mossi à l'est (2500-1800 Ma), séparés par la faille du Sassandra (Bessoles, 1977). La zone d'étude appartient aux régions du Poro et du Tchologo. Le sillon de Korhogo ou du Haut-Bandama, appartient au domaine Baoulé-Mossi qui couvre les deux tiers de la Côte d'Ivoire (Fig. 2). La société BHP Minerals, dans le cadre d'un partenariat avec la société pour le développement minier (SODEMI) a effectué un programme d'exploration minière dans la zone d'étude de 1988 à 1994. Cette mission révèlera l'existence d'unités volcanosédimentaires constituées d'une séquence de roches volcaniques basaltiques à andésitiques avec des intercalations de schistes, de tufs fins felsiques et des unités de cherts mineurs (BHP Minerals, 1994); (Adegoké M.B, 1996). Ces unités volcaniques contiennent de petites unités intrusives de granitoïdes calco-alcalins, de granitoïdes indifférenciés et de dykes doléritiques qui les recoupent 
localement. Ces auteurs montrent que la minéralisation aurifère dans cette zone est associée à des couloirs de cisaillements affectant principalement les unités birimiennes. En effet, la zone d'étude est encadrée de part et d'autre à l'ouest et à l'est par des granitoïdes. Ces lithologies observées dans zone d'étude sont affectées par un métamorphisme général de faciès schistes verts dominants à localement amphibolique. La figure 3 illustre la carte géologique de la zone d'étude.

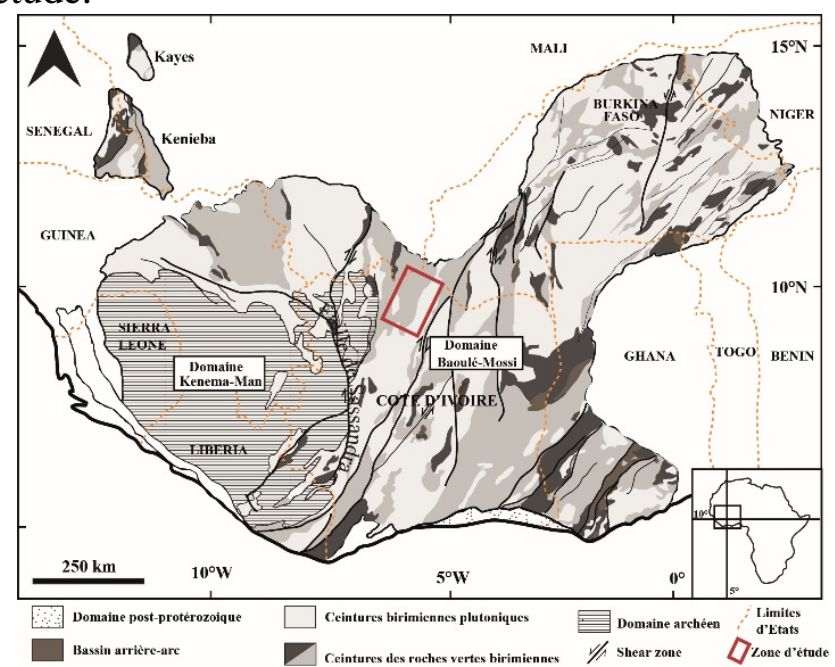

Figure 1. Carte géologique synthétique de la Dorsale de Man (modifiée, d’après Milési et al., 2004) et localisation de la zone d'étude

\section{Méthodologie}

Plusieurs échantillons de granitoïdes ont été sélectionnés au cours des différentes phases de terrain afin d'apporter plus de précision à la pétrographie macroscopique et également pour connaitre la signature géochimique des différentes roches. Les lames minces ont été confectionnées et étudiées au Laboratoire Géologie, Ressources Minérale et Energétique de l'UFR des Sciences de la Terre et des Ressources Minières (UFR STRM) de l'Université Félix Houphouët-Boigny d'Abidjan-Cocody. Sept échantillons de roches saines (cinq granodiorites et deux granites) ont été sélectionnés pour les analyses en éléments majeurs et en traces. Le broyage a été effectué par le Bureau Veritas à Abidjan (Côte d'Ivoire). Par la suite, les poudres ont été convoyées au Bureau Veritas Commodities Ltd (Canada), pour analyse chimique des éléments majeurs et en traces par la spectrométrie à émission atomique (ICP-AES) et par la spectrométrie de masse (ICP-MS). 


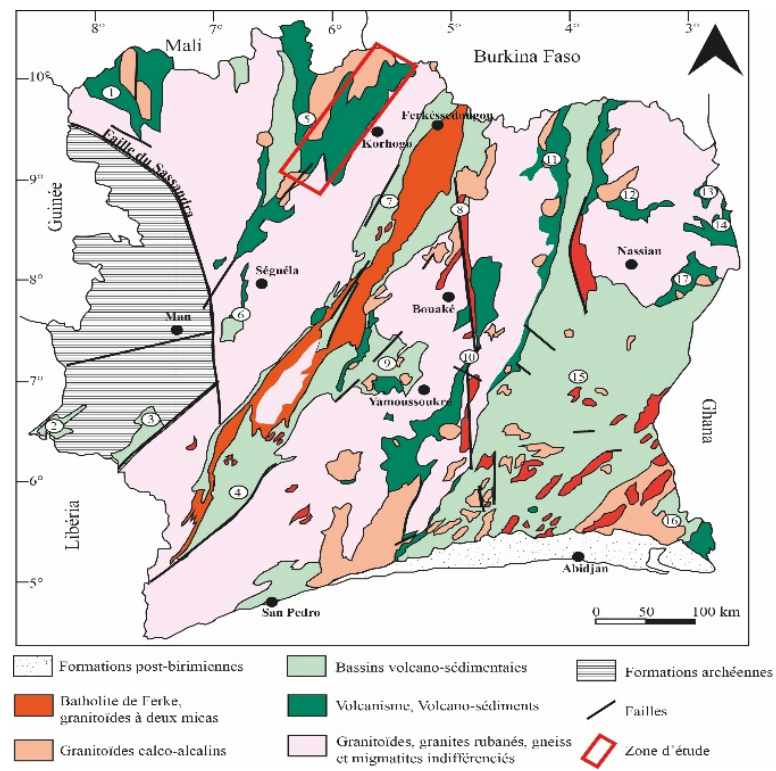

Figure 2. Schéma simplifié des ensembles géologiques en Côte-d’Ivoire, d’après la carte géologique au 1/1000000 de la Côte-d’Ivoire de Tagini (1972), modifiée et localisation de la zone d'étude.

1. sillon d'Odienné ; 2. sillon de Toulepleu ; 3. sillon de Guiglo ; 4. sillon de Soubré ; 5 sillon de Boundiali-Korhogo (Senoufo belt) ; 6 sillon de Séguela; 7. sillon de Ferké ; 8. sillon de Katiola ; 9. sillon de Bouaflé ; 10. sillon de BouakéEst ; 11. sillon de Téhini ; 12. sillon de Nassian-Nord ; 13. Sillon de Bouna-Sudest ; 14. sillon de Bondoukou-Nord; 15. sillon de Dimbokro-Abengourou; 16. Sillon d'Aboisso ; 17. sillon de Bondoukou

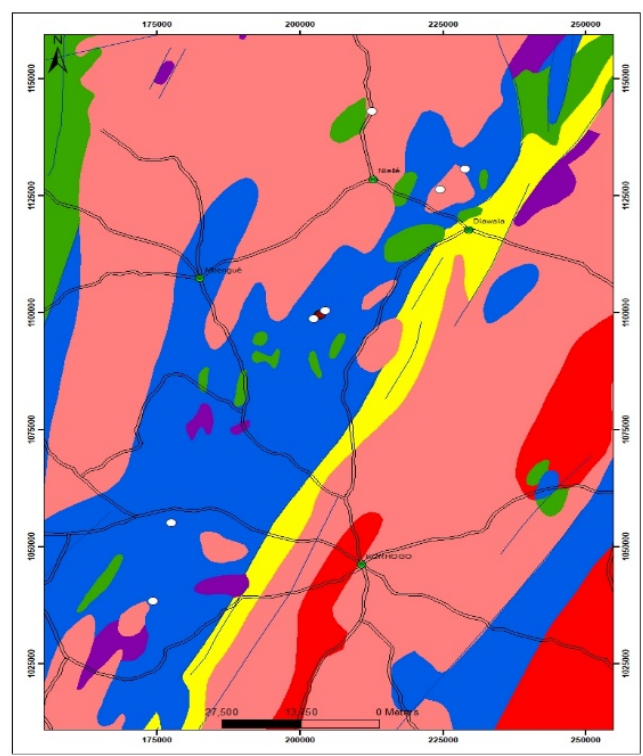

Figure 3. Carte de la zone d'étude modifiée d'après (Tagini, 1972) 


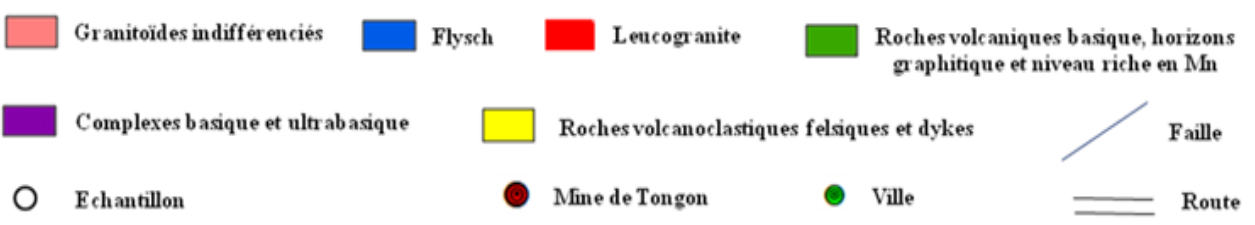

\section{Résultats}

\subsection{Pétrographie}

Les granitoïdes de la zone d'étude sont représentés généralement par les granodiorites et les granites.

\subsubsection{Granodiorite}

Ces roches ont été prélevées dans les localités de Niellé, Diawala et Fapoha. A l'affleurement, ces roches sont massives, mésocrates de texture grenue à grenue porphyroïde et en intrusion dans les métabasites (Figure 4A). Certains échantillons ont été prélevés généralement dans les zones d'orpaillages. On peut observer des cristaux de quartz, plagioclase, amphibole, biotite, des sulfures et des oxydes. Au microscope, ces roches sont généralement composées de quartz, de plagioclase, d'hornblende verte et de biotite (Figure 4 B-C-D). Le quartz se présente généralement sous forme de phénocristal avec une extinction roulante remarquable. Les cristaux de plagioclase, montrent également des phénocristaux qui sont souvent altérés et transformés en séricite, carbonates et épidote. Le plagioclase montre sur certaines sections une zonation magmatique. La hornblende verte est de la taille du grain moyen de la roche. La biotite, associée par endroit à la hornblende verte, montre des inclusions de zircons. Ces ferromagnésiens se déstabilisent souvent en chlorite et épidote. On note la présence de veinules de quartz-séricite associée à la chlorite et aux sulfures et oxydes.

\subsubsection{Granites}

Les granites affleurent dans presque toute la zone d'étude. Ces roches sont massives, généralement grenues, et en intrusion dans les métabasites (Figure 4E). Elles affleurent par endroit en bordure des cours d'eaux et sont constituées de cristaux de quartz, de feldspaths et de biotites. Ces granites sont parcourus par des filons de quartz. Au microscope les cristaux de plagioclase sont souvent altérés et transformés majoritairement en séricite et dans un degré moindre en épidote (Figure 4F). Le quartz quant à lui montre une extinction roulante remarquable. La biotite est présente avec certaines sections montrant un début de chloritisation. Cette dernière est plus abondante que la muscovite. On observe également la microcline, l'orthose ainsi que des minéraux opaques. 


\subsection{Géochimie}

Les analyses en éléments majeurs et en traces des granitoïdes du sillon de Korhogo sont résumées dans le tableau I.

\subsubsection{Eléments majeurs}

Les teneurs en éléments majeurs des granitoïdes du sillon de Korhogo ont des compositions de granites (4) et de granodiorites (2) sur le diagramme de Middlemost (1994) (Fig. 5).

Les granodiorites présentent des teneurs en $\mathrm{SiO}_{2}$ qui varient de 65,13 à 67,84\%. $\mathrm{MgO}$ varie de 1,37 à 2,95\% avec $(\mathrm{Mg} \#)=44,31-59,06 ; \mathrm{Fe}_{2} \mathrm{O}_{3}$ entre 3,80 et $5,47 \% ; \mathrm{Al}_{2} \mathrm{O}_{3}$ varie de 9,55 à $15,77 \%$ et $\mathrm{CaO}$ varie de 3,18 à $11,35 \%$. Les teneurs en $\mathrm{Na}_{2} \mathrm{O}$ et en $\mathrm{K}_{2} \mathrm{O}$ varient respectivement de 2,72 à 4,93\% et de 0,61 à $3,21 \%$. MnO est compris entre 0,07 et $0,10 \%$ et $\mathrm{TiO}_{2}$ varie de 0,40 à 0,57\%. Les diagrammes de Shand (1922) et de Irvine et Baragar. (1977) permettent de dire que ces roches sont métalumineux et calco-alcalines (Fig. 6 et 7).
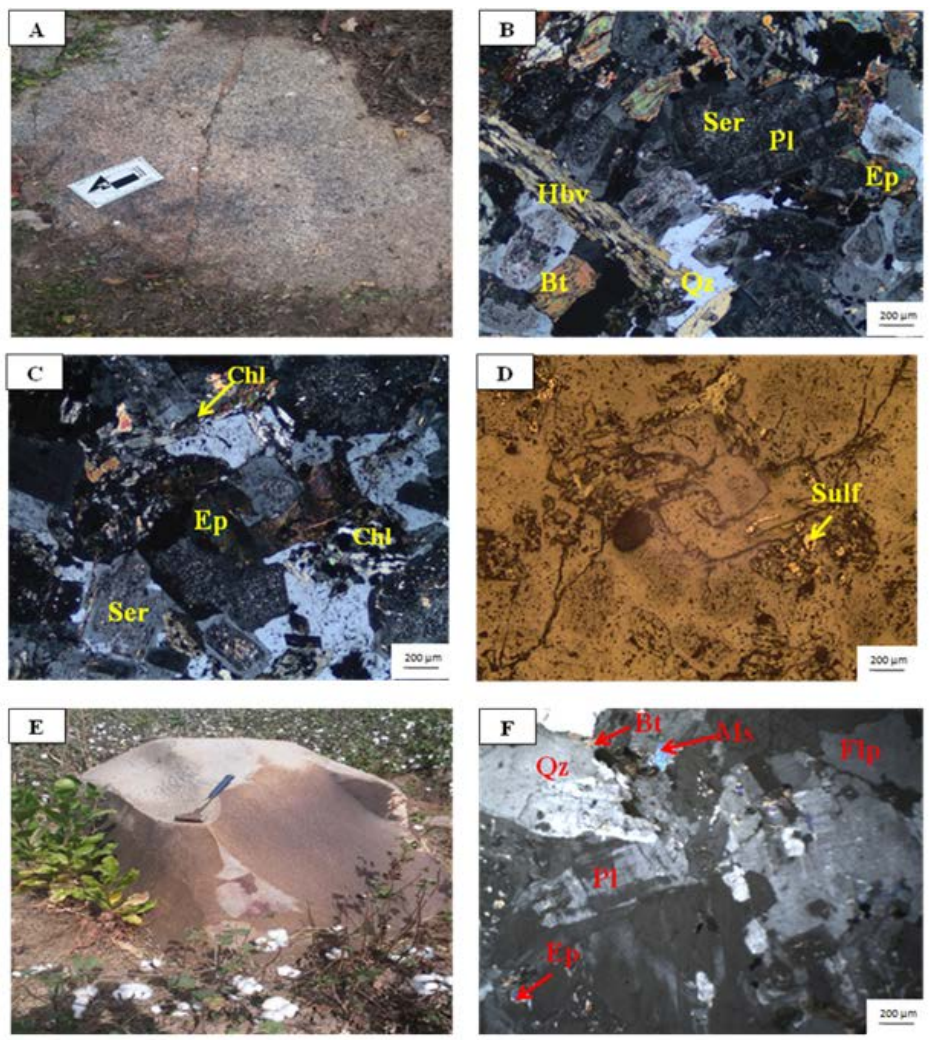

Figure 4. Aspects macroscopiques et microscopiques des granitoïdes du sillon de Korhogo.

A-B-C-D : Aspects macroscopique et microscopiques des granodiorites ; E-F : Aspects macroscopique et microscopique des granites. $\mathrm{Hb}-\mathrm{V}$ : Hornblende verte ; Bt : Biotite : Flp : Feldspaths ; Pl: Plagioclase ; Ms : Muscovite ; Qz : Quartz ; Ep : Epidote ; Chl : Chlorite ; Sulf : Sulfure 
Les granites sont caractérisés par des teneurs en $\mathrm{SiO}_{2}$ de 72,27 à 74,42 $\%$. $\mathrm{MgO}$ varie de 0,27 à $0,52 \%$ avec $(\mathrm{Mg} \#)=32,40-35,06 ; \mathrm{Fe}_{2} \mathrm{O}_{3}$ est compris entre 1,25 et $2,13 \% ; \mathrm{Al}_{2} \mathrm{O}_{3}$ est de $14,78 \%$ et $\mathrm{CaO}$ varie de 1,14 à $1,63 \%$. Les teneurs en $\mathrm{Na}_{2} \mathrm{O}$ varient de 4,65 à $5,37 \% ; \mathrm{K}_{2} \mathrm{O}$ est compris entre 2,14 et $4,03 \% ; \mathrm{MnO}$ et $\mathrm{TiO}_{2}$ varient respectivement de 0,04 à $0,05 \%$ et 0,09 à 0,31\%. Les diagrammes de Shand (1922) et de Irvine et Baragar. (1977) permettent de dire que les granites sont peralumineux et calco-alcalins (Fig. 6 et 7).

\begin{tabular}{|c|c|c|c|c|c|c|c|c|c|c|c|c|c|c|}
\hline & $\begin{array}{c}\text { Echantillon/Eléments } \\
\text { majeurs (\%) }\end{array}$ & $\mathrm{SiO} 2$ & $\mathrm{~A} 12 \mathrm{O} 3$ & $\mathrm{Fe} 2 \mathrm{O} 3$ & $\mathrm{CaO}$ & $\mathrm{MgO}$ & $\mathrm{Na} 2 \mathrm{O}$ & $\mathrm{K} 2 \mathrm{O}$ & $\mathrm{MnO}$ & $\mathrm{TiO} 2$ & $\mathrm{P} 2 \mathrm{O} 5$ & $\mathrm{Cr} 2 \mathrm{O} 3$ & LOI & Total \\
\hline Granodiorite & DIA21 & 67.84 & 14.54 & 4.27 & 3.18 & 2.02 & 4.61 & 2.94 & 0.08 & 0.4 & 0.12 & 0.02 & 0.43 & 100 \\
\hline Granodiorite & DIA25 & 65.61 & 15.07 & 5.33 & 4.08 & 2.21 & 4.49 & 2.38 & 0.09 & 0.57 & 0.17 & 0.02 & 0.62 & 100 \\
\hline Granodiorite & N02-1 & 67.5 & 9.55 & 5.47 & 11.35 & 2.12 & 2.72 & 0.61 & 0.1 & 0.46 & 0.12 & 0.03 & 4.36 & 100 \\
\hline Granodiorite & N010 & 65.13 & 15.11 & 4.5 & 4.1 & 2.95 & 4.26 & 3.21 & 0.08 & 0.48 & 0.17 & 0.04 & 1.65 & 100 \\
\hline Granite & DIA22-3 & 72.27 & 14.78 & 2.13 & 1.14 & 0.52 & 4.65 & 4.03 & 0.05 & 0.31 & 0.11 & 0.01 & 0.56 & 100 \\
\hline \multirow[t]{2}{*}{ Granite } & FAP18 & 74.42 & 14.78 & 1.25 & 1.63 & 0.27 & 5.37 & 2.14 & 0.04 & 0.09 & 0.02 & 0.02 & 0.65 & 100 \\
\hline & $\begin{array}{c}\text { Echantillon/Eléments } \\
\text { en traces (ppm) }\end{array}$ & $\mathrm{Ba}$ & $\mathrm{Be}$ & Co & $\mathrm{Cs}$ & $\mathrm{Ga}$ & $\mathrm{Hf}$ & $\mathrm{Nb}$ & $\mathrm{Rb}$ & $\mathrm{Sn}$ & $\mathrm{Sr}$ & $\mathrm{Ta}$ & Th & \\
\hline Granodiorite & DIA21 & 572 & 2 & 11.8 & 0.4 & 15.3 & 3.2 & 3.5 & 67.4 & $<1$ & 515.9 & 0.4 & 4.6 & \\
\hline Granodiorite & DIA25 & 516 & 1 & 12.2 & 1 & 17.1 & 3.8 & 4.1 & 63.1 & $<1$ & 645.1 & 0.4 & 3.9 & \\
\hline Granodiorite & N02-1 & 269 & 2 & 11.4 & 0.5 & 14.1 & 2.8 & 4.2 & 13.3 & $<1$ & 486.9 & 0.3 & 2.7 & \\
\hline Granodiorite & N010 & 824 & 2 & 14.4 & 2.7 & 16.8 & 3.8 & 5.7 & 64 & $<1$ & 713.9 & 0.5 & 6.7 & \\
\hline Granite & DIA22-3 & 976 & 1 & 6.4 & 2.4 & 15.9 & 4 & 6 & 120.3 & $<1$ & 558.5 & 0.7 & 8 & \\
\hline \multirow[t]{2}{*}{ Granite } & FAP18 & 981 & 3 & 1.2 & 0.6 & 13 & 1.5 & 3 & 42.4 & $<1$ & 697.5 & 0.2 & 0.4 & \\
\hline & $\begin{array}{c}\text { Echantillon/Eléments } \\
\text { en traces (ppm) }\end{array}$ & $\mathrm{U}$ & $\mathrm{V}$ & $\mathrm{W}$ & $\mathrm{Zr}$ & $\mathrm{Y}$ & La & $\mathrm{Ce}$ & $\operatorname{Pr}$ & $\mathrm{Nd}$ & $\mathrm{Sm}$ & $\mathrm{Eu}$ & $\mathrm{Gd}$ & \\
\hline Granodiorite & DIA21 & 1.8 & 86 & 0.7 & 113.2 & 41.2 & 23.5 & 36.5 & 4.95 & 18.8 & 3.46 & 0.99 & 4.14 & \\
\hline Granodiorite & DIA25 & 1.2 & 104 & $<0.5$ & 138.3 & 13.9 & 19 & 37.5 & 4.26 & 15.6 & 3.39 & 0.93 & 3.04 & \\
\hline Granodiorite & N02-1 & 0.6 & 63 & 22.4 & 98.3 & 13.5 & 20.9 & 39.9 & 4.67 & 17.8 & 3.79 & 1.79 & 3.44 & \\
\hline Granodiorite & N010 & 2.9 & 78 & 9.4 & 141 & 9.7 & 32 & 65.2 & 7.2 & 27.8 & 4.38 & 1.22 & 3.16 & \\
\hline Granite & DIA22-3 & 3.1 & 27 & 0.5 & 168.6 & 20 & 66.3 & 80.4 & 15.19 & 55.4 & 8.61 & 2.17 & 6.44 & \\
\hline \multirow[t]{2}{*}{ Granite } & FAP18 & 0.1 & $<8$ & $<0.5$ & 47.2 & 3.4 & 3.7 & 6.5 & 0.74 & 3.2 & 0.5 & 0.16 & 0.4 & \\
\hline & $\begin{array}{c}\text { Echantillon/Eléments } \\
\text { en traces (ppm) }\end{array}$ & $\mathrm{Tb}$ & $\mathrm{Ni}$ & As & $\mathrm{Cd}$ & $\mathrm{Sb}$ & $\mathrm{Bi}$ & $\mathrm{Ag}$ & $\mathrm{Au}$ & $\mathrm{Hg}$ & T1 & $\mathrm{Se}$ & & \\
\hline Granodiorite & DIA21 & 0.63 & 9.1 & 2.8 & $<0.1$ & $<0.1$ & $<0.1$ & $<0.1$ & 2.9 & $<0.01$ & $<0.1$ & $<0.5$ & & \\
\hline Granodiorite & DIA25 & 0.45 & 9.4 & 2.9 & $<0.1$ & $<0.1$ & $<0.1$ & $<0.1$ & 1.7 & $<0.01$ & $<0.1$ & $<0.5$ & & \\
\hline Granodiorite & N02-1 & 0.47 & 15.4 & 8.6 & $<0.1$ & 0.4 & $<0.1$ & 0.1 & 5.2 & 0.01 & $<0.1$ & $<0.5$ & & \\
\hline Granodiorite & N010 & 0.39 & 43.1 & 15.9 & $<0.1$ & 0.1 & $<0.1$ & $<0.1$ & $<0.5$ & $<0.01$ & 0.1 & $<0.5$ & & \\
\hline Granite & DIA22-3 & 0.77 & 9.8 & 0.9 & $<0.1$ & $<0.1$ & $<0.1$ & $<0.1$ & 1.6 & $<0.01$ & 0.1 & $<0.5$ & & \\
\hline Granite & FAP18 & 0.07 & 1.3 & 1 & $<0.1$ & $<0.1$ & $<0.1$ & $<0.1$ & 0.7 & $<0.01$ & $<0.1$ & $<0.5$ & & \\
\hline
\end{tabular}

\subsubsection{Eléments en traces}

Les compositions en éléments en traces des granitoïdes du sillon de Korhogo sont présentées dans le tableau I.

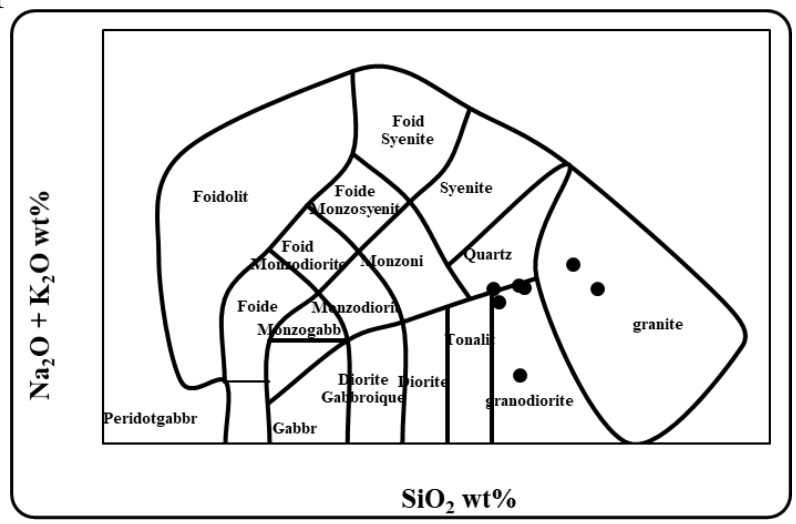

Figure 5. Diagramme de classification de Middlemost (1994) appliqué aux granitoïdes du sillon de Korhogo 


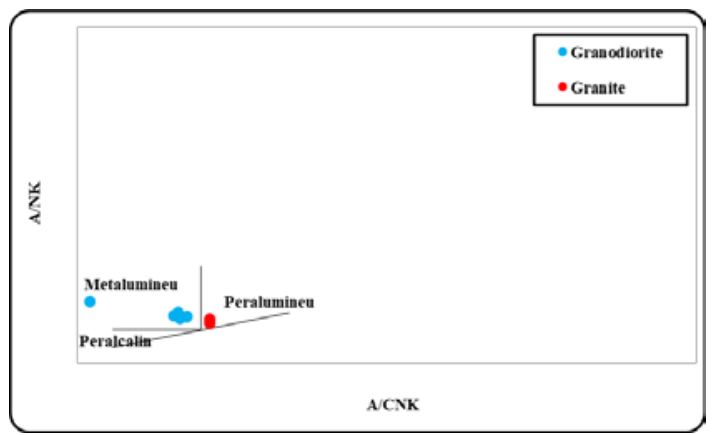

Figure 6. Diagramme de Shand (1922) appliqué aux granitoïdes du sillon de Korhogo

Figure 7. Diagramme AFM (Ivrine et Baragar, 1971) appliqué aux granitoïdes du sillon de Korhogo

La somme des terres rares ( $\Sigma \mathrm{REE})$ des granodiorites sont comprises entre 90,62 ppm et 145,94 ppm. Les spectres de terres rares des granodiorites normalisés à la chondrite montrent un enrichissement modéré à fort en LREE avec des taux de fractionnement de : $\left[(\mathrm{La} / \mathrm{Sm})_{N}=3,36-4,45\right.$ et $(\mathrm{La} / \mathrm{Yb})_{N}=$ 7,29-20,92] (Figure8). Ces spectres présentent également un appauvrissement en HREE $\left.(\mathrm{Gd} / \mathrm{Yb})_{N}=1,56-2,51\right)$.

Les anomalies négatives à légèrement positives en europium (Eu/Eu* $=0,81-1,53)$ observées seraient dues à une divergence dans l'évolution géochimique de ces roches. A cela s'ajoutent des anomalies négatives à quasiment nulles en cérium $\left(\mathrm{Ce} / \mathrm{Ce}^{*}=0,80-1,01\right)$.

La somme des terres rares ( $\mathrm{REE})$ des granites sont généralement comprises entre 16,72 ppm et 243,58 ppm. Les spectres des terres rares de ces roches normalisés aux chondrites selon Sun \& McDonough (1989), montrent un enrichissement en LREE avec un taux de fractionnement modéré à élevé: $\left[(\mathrm{La} / \mathrm{Sm})_{N}=4,51-4,69\right.$ et $\left.(\mathrm{La} / \mathrm{Yb})_{N}=7,63-26,53\right]$, à raison de 2 à 210 fois la teneur chondritique (Fig. 8). Comparativement au LREE, il y a une tendance d'appauvrissement en HREE [ $\left.(\mathrm{Gd} / \mathrm{Yb})_{N}=1,00-3,13\right]$, à raison de 1 à 25 fois la teneur chondritique. Ces roches présentent généralement une anomalie légèrement négative à positive en europium $\left(\mathrm{Eu} / \mathrm{Eu}^{*}=0,90-1,11\right)$, ainsi que des anomalies négatives en cérium $\left(\mathrm{Ce} / \mathrm{Ce}^{*}=0,60-0,92\right)$. L'anomalie négative 
en Ce est commune aux magmas d'arcs modernes mais peut également résulter des altérations post-magmatiques tels que la circulation dominante des fluides hydrothermaux (Abouchami et al., 1990 ; Sylvester et Attoh, 1992).

Les spectres multiéléments des granitoïdes normalisés au manteau primitif (Figure 9), montrent des enrichissements significatifs en LILEs (Cs, $\mathrm{Rb}, \mathrm{Ba}$ et $\mathrm{K}$ ) associée à une anomalie négative générale en $\mathrm{Nb}, \mathrm{Ta}, \mathrm{P}, \mathrm{Ti}$ et $\mathrm{V}$. Les dispositifs géochimiques tels que l'enrichissement en $\mathrm{Ba}$ et $\mathrm{Sr}$ et l'appauvrissement en $\mathrm{Nb}$, Ta, et Ti sont des caractéristiques typiques des zones de subduction. Ces spectres présentent également dans l'ensemble un enrichissement modéré des HFSE (Hf, Zr).

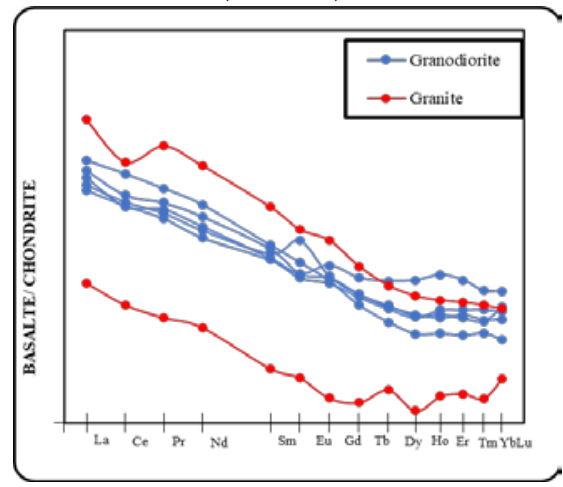

Figure 8. Spectres des terres rares normalisées aux chondrites appliqués aux granitoïdes du sillon de Korhogo

\subsubsection{Contexte géodynamique}

Les analyses chimiques des granitoïdes du sillon de Korhogo, reportées dans le diagramme ternaire Rb Rb-Hf-Ta de Harris et al. (1986), montrent une affinité des granitoïdes aux roches des arcs volcaniques (Figure 10). Dans le diagramme $\mathrm{La} / \mathrm{Yb}-\mathrm{Yb}$ de Martin (1986) de la figure 11, les granitoïdes se situent généralement dans le champ commun aux TTG archéennes et des roches des arcs insulaires. Cette position de ces roches montre qu'elles ont une certaine affinité aux TTG archéennes. Cela pourrait indiquer une source mixte lors de la mise en place de ces formations.

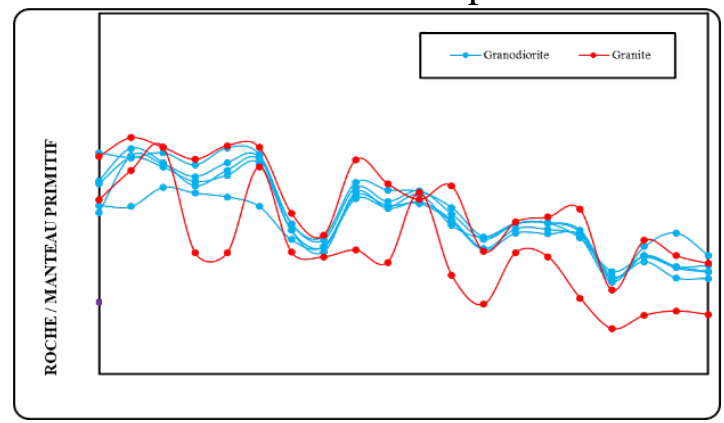

Figure 9. Spectres multi-éléments normalisés au manteau primitif appliqués aux granitoïdes du sillon de Korhogo 


\section{Discussion}

Les données pétrographiques recueillies sur les granitoïdes du sillon de Korhogo indiquent qu'ils ont des compositions de granites et de granodiorites. Ces granitoïdes sont affectés par une intense altération pervasive et fissurale témoignant ainsi de l'intensité de l'altération hydrothermale dans le sillon de Korhogo. En effet, la plupart des minéraux primaires montrent des transformations en minéraux secondaires. Ces minéraux s’altèrent généralement en carbonates, chlorite, séricite et épidote. A cela s'ajoute également une silicification, une oxydation ainsi qu'une sulfudation. L'hydrothermalisme est marqué par la formation de veines quartzofeldspathiques associées à des sulfures, des carbonates et de la séricite. Ces types d'altérations s'apparentent généralement à celles des gisements d'Agbahou (Houssou, 2013), de Bonikro (Ouattara, 2015), de Dougbafla (Ouattara, 2018) et au prospect de Bobosso de la région de Dabakala (Gnanzou, 2014).

L'ensemble de ces roches a été affecté par le métamorphisme régional de faciès schistes verts. Ces conditions métamorphiques de faciès généralement schistes verts sont connus dans les sillons birimiens OuestAfricains (Hirst, 1942 ; Leube et al., 1990 ; Hirdes et al., 1992 ; Taylor et al., 1992 ; Sylla et Ngom, 1997 ; Bourges et al., 1998 ; Houssou, 2013, Coulibaly, 2018). Les granitoïdes du sillon de Korhogo contiennent du microcline tout comme ceux du sillon de Toumodi-Fètêkro (Coulibaly, 2018) et du gisement de Bonikro (Ouattara., 2015). En effet, pour Casanova (1973), cette microclinitisation atteste d'un apport tardif d'eau et même postérieur à la mise en place des roches qui s'est effectuée entre 400 et $500^{\circ} \mathrm{C}$.

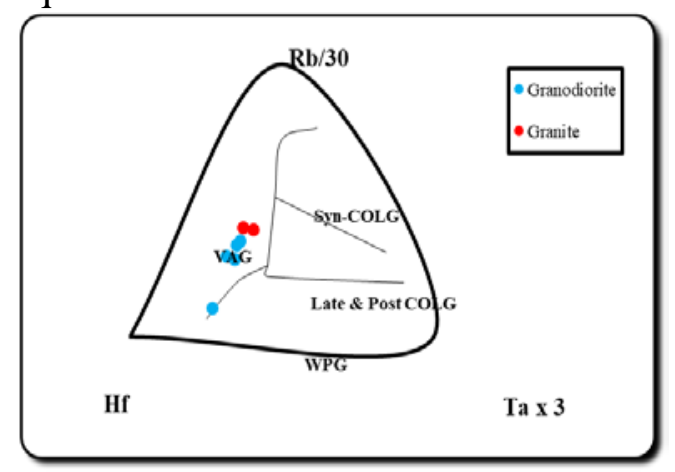

Figure 10. Diagramme ternaire Rb-Hf-Ta*3 de Harris et al. (1986) appliqué aux granitoïdes du sillon de Korhogo 


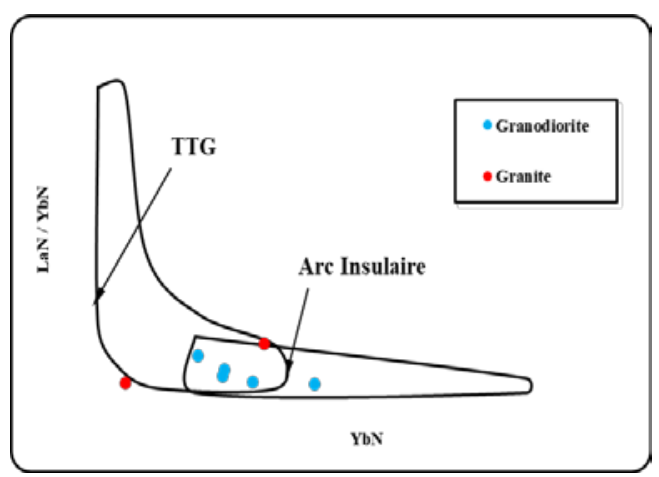

Figure 11. Diagramme $\mathrm{La}_{N} / \mathrm{Yb}_{N}$ en fonction de $\mathrm{Yb}_{N}$ de Martin (1986) appliqué aux granitoïdes du sillon de Korhogo

Les granitoïdes du sillon de Korhogo sont fortement calco-alcalins, dominés par le potassium et ont des compositions de granites et de granodiorite tout comme dans le gisement de Bonikro tout comme dans le gisement de Bonikro (Ouattara, 2015), sur le pluton de Dabakala (Doumbia et al., 1998), dans les granitoïdes de Bouaké (Casanova., 1973).

Les données géochimiques montrent que les granitoïdes du sillon de Korhogo ont des taux de $\mathrm{TiO}_{2}$ inférieurs à $2 \%$. En effet, selon Pearce et Cann (1973), les échantillons de roches ayant de faibles taux de $\mathrm{TiO}_{2}(<2 \%)$ correspondent aux roches des arcs magmatiques (Pearce et Cann, 1973), mais sont différents des basaltes intra-plaques, qui possèdent souvent des taux de $\mathrm{TiO}_{2}$ élevés ( $>2 \%$ ). Cela se confirme également à travers les arachnogrammes de ces roches normalisées au manteau primitif. En effet, ces spectres montrent généralement un enrichissement important en LILE (Cs, Ba, Rb, K) et une nette anomalie de $\mathrm{Nb}$-Ta indiquant clairement un environnement de subduction. Cependant, le Nb et le Ta sont également extrêmement sensibles à la contamination crustale. Les magmas dérivés du manteau qui pourraient être contaminés par les roches dans la croûte continentale pendant leur montée en surface ont des spectres multiéléments marqués par une anomalie négative de Nb-Ta (Thompson et al., 1983 ; Wilson, 1989). Les rapports de $(\mathrm{La} / \mathrm{Yb})_{N}$ ainsi que les taux de $\mathrm{Yb}_{N}$ des granitoïdes compris respectivement entre 7,29 et 20,92 et entre 1,54 ppm et 10,24 ppm évoquent une possible contamination crustale lors de la mise en place des roches du sillon de Korhogo. De telles observations ont été mises en évidence en Côte d'Ivoire par Coulibaly (2018) dans la partie sud du sillon birimien de Toumodi-Fètêkro et par Téha (2019) dans le Sud-Ouest du bassin de la Comoé et en Algérie par Peucat et al. (2005) dans le massif d'Eglab. A cela s'ajoute le fait que les granitoïdes analysés du sillon de Korhogo sont métalumineux à peralumineux et de type I (Figure 6 et 12). 


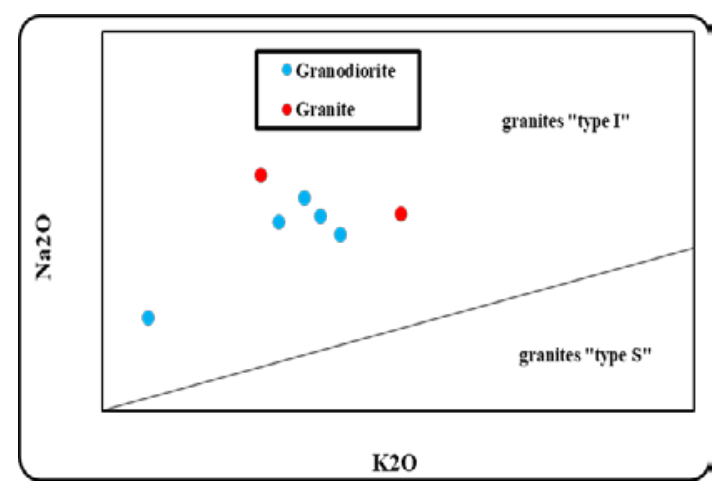

Figure 12. Diagramme de Chappell \& White (1974) appliqué aux granitoïdes du sillon de Korhogo

En effet, selon Chappell et White (1974) les granitoïdes de type I ont une origine ignée et proviendraient d'une part de la fusion du manteau et d'autre part de la fusion de roches cristallines de la croûte continentale. Ces observations faites, ont déjà été mises en évidence dans les sillons birimiens ivoiriens (Gnanzou, 2014 ; Ouattara, 2015 ; Coulibaly, 2018 ; Téha, 2019) et ailleurs dans le craton Ouest-africain (Peucat et al., 2005 ; Baratoux et al., 2011). Les travaux réalisés dans le sillon de Korhogo confirment la présence de l'héritage archéen, évoqué par endroits dans les formations juvéniles birimiennes (Kouamelan, 1996 ; Peucat et al. (2005) ; Coulibaly, 2018).

\section{Conclusion}

Les données pétrographiques recueillies dans les granitoïdes du sillon de Korhogo sont des granites et des granodiorites. Les granodiorites sont généralement constituées de quartz, de plagioclase, d'hornblende verte et de biotite, de chlorite, épidote, de sulfures et oxydes. Les granites quant à eux sont composés de plagioclase, d'orthose, de quartz, de biotite, de muscovite, de microcline, de chlorite, de séricite, d'épidote et de minéraux opaques. Ces granitoïdes sont affectés par une intense altération pervasive et fissurale témoignant ainsi de l'intensité de l'altération hydrothermale dans le sillon de Korhogo. L'étude détaillée des lames minces a montré la présence de minéraux symptomatiques de basse pression et de faible température tels que l'épidote, la chlorite, la séricite témoignant que ces roches ont été affectées par le métamorphisme de faciès schiste vert.

Les données géochimiques montrent que les granitoïdes du sillon de Korhogo sont calco-alcalins et métalumineux à peralumineux. Ils sont généralement enrichis en LREE et sont généralement de Type «I ». Les anomalies négatives à quasiment nulles en cérium observées sont communes aux magmas d'arcs modernes mais peut également résulter d'altérations postmagmatiques tels que la circulation pervasive de fluides hydrothermaux. Les 
taux de $\mathrm{TiO}_{2}$ inférieurs à $2 \%$ observés montrent que ces roches ont été mises en place dans un contexte d'arc magmatique. Cela se confirme à travers les arachnogrammes normalisés aux au manteau primitif qui montrent généralement un enrichissement important en LILE (Cs, Ba, Rb, K) et une nette anomalie de Nb-Ta indiquant clairement un environnement de subduction. Cependant les le comportement de certains éléments majeurs et en trace permet d'évoquer une possible contamination crustale lors de la mise en place des roches du sillon de Korhogo.

\section{References:}

1. Abouchami W., Boher M., Michard A., Albarede F. (1990). A Major 2,1Ga Event of Mafic Magmatism in West Africa: An Early Stage of Crustal Accretion. J. Geophys. Res., 95 : 17605-17629.

2. Adegoke M. B. (1996). Rapport final d'activités minières sur les permis de sénoufo Nord, sénoufo Sud et Niellé dans le Nord de la Côte d'Ivoire, rap int. SODEMI/BHP Minerals. 233p.

3. Baratoux L., Metelka V., Naba S., Jessell M.W., Gregoire M., Ganne J. (2011). Juvenile Paleoproterozoic crust evolution during the Eburnean orogeny ( 2.2-2.0 Ga), western Burkina Faso. Precambrian Res. 191, 18-45.

4. Bessoles B. (1977). Géologie de l’Afrique. Vol 1 : le Craton Ouest Africain. Bur. Rech. Géol. Min. Mem. 88. 402 p.

5. Bourges F., Debat P., Tollon F., Munoz M., Ingles J. (1998). The geology of the Taparko gold deposit, Birimian greenstone belt, Burkina Faso, West Africa. Mineralium Deposita 33, 591-605.

6. Casanova R. (1973). Géochimie et minéralogie des granitoïdes Eburnéens de Côte d'Ivoire. Thèse de Doctorat d'Etat ès sciences, Université de Nice. 393p.

7. Chappell B.W., White A.J.R. (1974). Two contrasting granite types. Pacific Geology 8: 173-174.

8. Coulibaly I. (2018). Pétrographie des volcanites et plutonites de la partie Sud du sillon volcanosédimentaire de Toumodi-Fétèkro. Thèse Univ. Felix Houphouët-Boigny d'Abidjan. 218p.

9. Doumbia S., Pouclet A., Kouamelan A., Peucat J. J., Vidal M. (1998). Petrogenesis of juvenile-type Birimian (Paleoproterozoic) granitoïds in Central Côte d'Ivoire, West Africa: geochemistry and geochronology. Precambrian Research 87, 33-63.

10. Feybesse J.L. (2001). Géométrie et cinématique des fractures contrôlant la mise en place des veines et veinules de quartz de la mine d'or d'Angovia (Yaouré, Côte d'Ivoire). Compagnie Minière d'Afrique, Rapport interne, non publié. 
11. Gbamélé K. (2012). Facteurs gouvernant le choix d'un permis de recherche minière pour l'or. Cas de la Côte d'Ivoire. Mém. Ing. des Tech., Ecole de Formation Cont. et de Perfect. des Cadres, INPHB, Yamoussoukro, $77 \mathrm{p}$.

12. Gnanzou A. (2014). Etude des séries volcano-sédimentaires de la région de Dabakala (Nord-Est de la Côte d'Ivoire) : genèse et évolution magmatique. Contribution à la connaissance de la minéralisation aurifère de Bobosso dans la série de la Haute-Comoé. Thèse de doctorat, Univ. Paris-Sud Orsay, France et Univ. Félix Houphouët-Boigny, 303p.

13. Harris N.B.W., Pearce J.A., Tindle A.G. (1986). Geochemical characteristics of collision-zone magmatism. In: M.P. Cowardand A.C. Reis (Editeurs), Collision tectonics, Geol. Soc. London. Spec. Publ., 19: 67-81.

14. Hirdes W., Davis D.W. (2002). U-Pb geochronology of Paleoproterozoic rocks in the southern part of the Kedougou-Kéniéba inlier, Senegal, West Africa: Evidence for diachronous accretionary development of the Eburnean province: Precambrian Research, v. 118: 83-99.

15. Hirdes W., Davis D.W., Eisenlohr N.N. (1992). Reassessment of Proterozoic granitoid ages in Ghana on the basis of $\mathrm{U} / \mathrm{Pb}$ zircon and monazite dating. Precambrian Res., 56 : 89-96.

16. Hirst T. (1942). The geology of the Konongo gold belt and surrounding country. Gold Coast Geol. Surv. Bull. 14.

17. Houssou N.N. (2013). Etudes pétrologique, structurale et métallogénique du gisement aurifère d'Agbahou, Divo, Côte d'Ivoire. Doctorat, Univ. Félix Houphouët -Boigny, 177p.

18. Irvine T N., Baragar W. R. A. (1971). A guide to the chemical classification of the common volcanic rocks. Can. J. Earth Sci. 8: 523548.

19. Kouamelan A.N. (1996). Géochronologie et Géochimie des formations archéennes et protérozoïques de la dorsale de Man en Côte d'Ivoire: Implication pour la transition Archéen-Protérozoïque. Mémoire Géoscience n 73 Rennes. 293 p.

20. Leube A., Hirdes W., Mauer R., Kesse G.O. (1990). The early Proterozoic Birimian supergroup of Ghana and some aspects of its associated gold mineralization. Precambrian Research 46, pp. 139165.

21. Martin H. (1986). Effect of steeper Archaean geothermal gradient on geochemistry of subduction-zone magmas. Geology, 14: 753-756.

22. McDonough W.F., Sun S., Ringwood A.E., Jagoutz E., Hofmann A.W. (1992). K, Rb and Cs in the earth and moon and the evolution 
of the earth's mantle. Geochim. Cosmochim. Acta, Ross Taylor Symposium Volume.

23. Middlemost E. A. K. (1994). Naming materials in the magma/igneous rock system. Earth Science Reviews, 37 (3-4), pp. 215-224.

24. Milési J.P., Feybesse J.L., Ledru P., Dommanget A., Ouédraogo M.F., Marcoux E., Prost A.E., Vinchon C., Sylvain J.P., Johan V., Tegyey M., Calvez J.Y., Lagny Ph. (1989). Les minéralisations aurifères de l'Afrique de l'Ouest. Leur évolution lithostructurale au Protérozoïque inférieur. Chron. Rech. min., Fr., 497 : 3-98.

25. Milesi J.P., Ledru P., Feybesse J.L., Dommanget A. and Marcoux E. (1992). Early Proterozoïc ore deposits and tectonics of the Birimian orogenic belt, West Africa. Precambrian Research 58, I ssues 1-4, pp. 305-344.

26. Milesi J.P., Feybesse J. L., Pinna P., Deschamps Y., Kampunzu H., Muhongo S.,

27. Lescuyer .J.L., Le Goff E., Delor C. and Billa M. (2004). Geological map of Africa 1:10 000 000. SIG Afrique project, BRGM Orléans.

28. Morel B. and Alinat M. (1993). Géologie, pétrologie et géochimie des syénites de Ninakri: comparaison avec d'autres massifs syénitiques anorogéniques de Côte d'Ivoire et d'Afrique de l'Ouest. J. Afr. Earth Sci 17: 213-223.

29. Naba S., Lompo M., Débat P., Bouchez J.L and Béziat D. (2004). Structure and emplacement model for late-orogenic Paleoproterozoic granitoids: the Tenkodogo- Yamba elongate pluton (Eastern Burkina Faso). Journal of African Earth Sciences 38: 41-57.

30. Ouattara S. (2018). Le gisement de Dougbafla-Bandama (Sud du sillon birimien de Fêttêkro, Oumé, Côte d'Ivoire): Pétrographie, déformation, géochimie et métallogénie. Doctorat, Univ. Felix Houphouet-Boigny, $252 \mathrm{p}$.

31. Ouattara Z. (2015). Caractères lithostratigraphique, structural, géochimique et métallogénique du gisement d'or de Bonikro, sillon birimien de Fettekro, centre-sud de la Côte d'Ivoire. Doctorat, Univ. Felix Houphouet-Boigny, 256 p.

32. Pearce J.A., Cann J.R. (1973). Tectonic setting of basic volcanic rocks determined using trace element analyses. Earth Planet. Sci.Lett., 19 :290-300.

33. Peucat J.J., Capdevila R., Drareni A., Mahdjoub Y., Kahoui M. (2005). The Eglab massif in the West African Craton (Algeria), an original segment of the Eburnean orogenic belt: petrology, geochemistry and geochronology. Precambr. Res. 136, 309-352. 
34. Shand S.J. (1922). The problem of the alkaline rocks. Proceedings of the Geological Society of South Africa 25: 19-33.

35. Sylla M., Ngom P. M. (1997). Le gisement d'or de Sabodala (Sénégal Oriental): une Minéralisation filonienne d'origine hydrothermale remobilisée par une tectonique cisaillante. J.of Afr. Earth Sces, 25, No. 2, pp. 183-192.

36. Sylvester P.J., Attoh K. (1992). Lithostratigraphy and composition of $2.1 \mathrm{Ga}$ greenstone belts of the West African craton and their bearing on crustal evolution and Archean-Proterozoic boundary.J. Geol. 100, 377-393.

37. Tagini B. (1972). Carte géologique de la Côte d'Ivoire à l'échelle 1/2 000 000. SODEMI-ORSTOM-Université d'Abidjan.

38. Tapsoba B., Ching-Hua L., Bor-Ming J., Sun-Lin C., Wenmenga U. and Yoshiyuki L. (2013). Chemical and Sr-Nd isotopic compositions and zircon $\mathrm{U}-\mathrm{Pb}$ ages of the Birimiangranitoids from NE Burkina Faso, West African Craton: Implications on the geodynamic setting and crustal evolution. Precambrian Research 224: 364-396.

39. Taylor P.N., Moorbath S., Leube A., Hirdes W. (1992). Early Proterozoic crustal evolution in the Birimian of Ghana: constraints from geochronology and isotope geochemistry. Precambrian Res., 56 : 97-111.

40. Téha K.R. (2019). Les Formations Éburnéennes du Sud-ouest du bassin de la Comoé et du secteur de Singrobo (Sud de la Côte d'Ivoire) : Pétrologie, Analyse structurale et Magmatisme associé. Thèse, Univ. Felix Houphouët -Boigny, 247 p.

41. Thompson R.N., Morrison M.A., Dickin A.P., Hendry G.L. (1983). Continental floodbasalts...arachnids rule OK? In: Hawkesworth, C.J., Norry, M.J. (Eds.), Continental Basalts and Mantle Xenoliths. Shiva Publishing, Chesire, UK : 158-185.

42. Turner P., Hall R. P., Hughes D. J., Whalley J. S. (1993). The sediment-dominated Boundiali-Bago supracrustal belt and neighbouring granitic rocks, northern Côte d'Ivoire, West Africa: a Tarkwaian connection. Journal of African Earth Sciences, Vol. 17, No. 1:1-11.

43. Wilson M. (1989). Igneous Petrogenesis. Unwin Hyman, London, 457 p.

44. Yacé I. (2002). Initiation à la géologie. L’exemple de la Côte d'Ivoire et de l'Afrique de l'Ouest. Edition CEDA, Abidjan, Côte d'Ivoire, 183p. 